\title{
AUTHOR INDEX \\ Volume 6 (2015)
}

Amdur, D., Dale, D., Borick, C. \& Rabe, B. G., Individual Discount Rates and Climate Change: Is Discount Rate Associated with Support for a Carbon Tax?

4 (2015) 1550018

Banerjee, O., Mahzab, M., Raihan, S. \& Islam, N., An Economy-Wide Analysis of Climate Change Impacts on Agriculture and Food Security in Bangladesh

1 (2015) 1550003

Bistline, J. E., Fat-Tailed Uncertainty, Learning, and Climate Policy

2 (2015) 1550009

Borick, C., see Amdur, D.

4 (2015) 1550018

1 (2015) 1550004

4 (2015) 1550018

Dale, D., see Amdur, D.

4 (2015) 1550017

Das, S., Temperature Increase, Labor Supply and Cost of Adaptation in Developing Economies: Evidence on Urban Workers in Informal Sectors

2 (2015) 1550007

$1(2015) 1550005$

Dinar, A., see Rahman, S. M.

Eriksson, M., The Role of the Forest in an Integrated Assessment Model of the Climate and the Economy

3 (2015) 1550011

Estrada, F. \& Tol, R. S. J., Toward Impact Functions for Stochastic Climate Change

$4(2015) 1550015$

4 (2015) 1550017

Guivarch, C., Monjon, S., Rozenberg, J. \& Vogt-Schilb, A., Would Climate Policy Improve the European Energy Security?

2 (2015) 1550008

Halimanjaya, A. \& Papyrakis, E., Donor Characteristics and the Allocation of Aid to Climate Mitigation Finance

3 (2015) 1550014

1 (2015) 1550001

4 (2015) 1550019

Heine, D., see Parry, I.

Heuson, C., Peters, W., Schwarze, R. \& Topp, A.-K., Voluntary International Climate Finance Under the Post-Kyoto Framework: The Strategic Consequences of Different Modes of Funding

3 (2015) 1550013

Honlonkou, A. N. \& Hassan, R. M., Developing Countries' Response to the Clean Development Mechanism Under Imperfect Information and Transaction Costs

Iqbal, K. \& Roy, P. K., Climate Change, Agriculture and Migration:

Evidence from Bangladesh 
Islam, N., see Banerjee, O.

1 (2015) 1550003

Koopman, J. F. L., Kuik, O., Tol, R. S. J. \& Brouwer, R., Water

Scarcity from Climate Change and Adaptation Response in an International River Basin Context

1 (2015) 1550004

Kuik, O., see Koopman, J. F. L.

1 (2015) 1550004

Larson, D. F., see Rahman, S. M.

1 (2015) 1550005

Mahzab, M., see Banerjee, O.

1 (2015) 1550003

McNeill, J., see Sajeewani, D.

3 (2015) 1550012

Monjon, S., see Guivarch, C.

2 (2015) 1550008

Palmer, K., see Paul, A.

4 (2015) 1550016

Papyrakis, E., see Halimanjaya, A.

3 (2015) 1550014

Parry, I., Veung, C. \& Heine, D., How Much Carbon Pricing is in Countries' Own Interests? The Critical Role of Co-Benefits

$4(2015) 1550019$

Paul, A., Palmer, K. \& Woerman, M., Incentives, Margins, and Cost Effectiveness in Comprehensive Climate Policy for the Power Sector

4 (2015) 1550016

Peters, W., see Heuson, C.

Poljak, D., see Seung, C. K.

3 (2015) 1550013

4 (2015) 1550017

4 (2015) 1550017

4 (2015) 1550018

Rabe, B. G., see Amdur, D.

$1(2015) 1550005$

Emissions Abatement Under the Clean Development Mechanism

1 (2015) 1550003

1 (2015) 1550002

Riddle, A., see Sedjo, R. A.

Rosendahl, K. E. \& Storrøsten, H. B., Allocation of Emission

Allowances: Impacts on Technology Investments

Roy, P. K., see Iqbal, K.

3 (2015) 1550010

2 (2015) 1550006

2 (2015) 1550008

Sajeewani, D., Siriwardana, M. \& McNeill, J., Household

Distributional and Revenue Recycling Effects of the Carbon Price in Australia

3 (2015) 1550012

3 (2015) 1550013

Sedjo, R. A., Sohngen, B. \& Riddle, A., Land Use Change, Carbon, and Bioenergy Reconsidered

$1(2015) 1550002$

Seung, C. K., Dalton, M. G., Punt, A. E., Poljak, D. \& Foy, R., Economic Impacts of Changes in an Alaska Crab Fishery from Ocean Acidification

4 (2015) 1550017

3 (2015) 1550012

1 (2015) 1550002

3 (2015) 1550010

Storrøsten, H. B., see Rosendahl, K. E.

Tian, H. \& Whalley, J., Developing Countries and the UNFCCC

Process: Some Simulations from an Armington Extended Climate Model

4 (2015) 1550020

Tol, R. S. J., see Estrada, F.

4 (2015) 1550015

Tol, R. S. J., see Koopman, J. F. L.

1 (2015) 1550004 
Topp, A.-K., see Heuson, C.

3 (2015) 1550013

Veung, C., see Parry, I.

4 (2015) 1550019

Vogt-Schilb, A., see Guivarch, C.

2 (2015) 1550008

Whalley, J., see Tian, $\mathrm{H}$.

4 (2015) 1550020

Woerman, M., see Paul, A.

4 (2015) 1550016 